\title{
Tratamento de Pacientes com Úlceras Isquêmicas Secundárias à Esclerose Sistêmica com N-Acetilcisteína Endovenosa
}

\section{Treatment of Patients with Isquemic Ulcers Secondary to Systemic Sclerosis with Intravenous N-Acetylcysteine}

\author{
Cristiane Kayser ${ }^{(1)}$, Karine Rodrigues da Luz $^{(2)}$, Livanio Ferreira da Rocha ${ }^{(3)}$, Luís Eduardo C. Andrade ${ }^{(4)}$
}

\section{RESUMO}

Os repetidos episódios de isquemia-reperfusão observados na esclerose sistêmica (ES) acarretam aumento na atividade de radicais livres, o que pode estar implicado nas anormalidades vasculares e inflamatórias descritas nessa enfermidade. A N-acetilcisteína sob forma endovenosa é uma potente droga antioxidante e, como tal, poderia ter efeito benéfico para o tratamento das lesões vasculares da ES. Relatamos o tratamento com $\mathrm{N}$-acetilcisteína endovenosa de três pacientes com diagnóstico de ES e com úlceras ativas de extremidades (dígitos ou artelhos). Dois pacientes apresentavam duas úlceras digitais e o terceiro paciente, três úlceras em artelhos no início do tratamento. Todos os pacientes apresentaram diminuição no diâmetro de pelo menos uma úlcera após o tratamento. Duas pacientes apresentaram cicatrização de uma úlcera. Esses resultados preliminares sugerem que a $\mathrm{N}$-acetilcisteína endovenosa parece ser uma boa opção terapêutica para o tratamento de úlceras de extremidades em pacientes com ES e justificam a elaboração de ensaios controlados duplo-cego com placebo.

Palavras-chave: esclerose sistêmica, úlceras isquêmicas, fenômeno de Raynaud, N-acetilcisteína.

\section{INTRODUÇÃO}

A esclerose sistêmica (ES) é uma doença reumática auto-imune de etiologia desconhecida, caracterizada por anormalidades imunológicas, acometimento vascular da microcirculação e deposição excessiva de colágeno na pele e órgãos internos, afetando particularmente o trato gastrintestinal, pulmão, coração e rins ${ }^{(1)}$.

Alterações vasculares são um evento central na patogênese da ES, sendo que anormalidades estruturais e

\begin{abstract}
The repetitive ischemic-reperfusion episodes in patients with systemic sclerosis $\left(S S_{c}\right)$ cause an increase in free radical activity, which may be implicated in the inflammatory and vascular lesions observed in this illness. Endovenous $\mathrm{N}$-acetylicysteine is a potent anti-oxidant agent and might be beneficial to the treatment of vascular events in SSc. This communication reports on the use of endovenous $N$-acetylcysteine in three $S S_{c}$ patients with active ulcers in fingers and toes. At baseline, two patients presented two finger ulcers each, and a third patient had three ulcers in the toes. All patients presented a decrease in the dimensions of at least one ulcer. Two patients presented complete healing of one ulcer. These preliminary results suggest that endovenous $\mathrm{N}$-acetylcysteine may be an efficient therapeutic option for extremity ulcers in SSc patients and support future randomized double blind placebo-controlled trials.
\end{abstract}

Keywords: systemic sclerosis, ischemic ulcers, Raynaud's phenomenon, $\mathrm{N}$-acetylcysteine.

funcionais da microcirculação podem ser detectadas precocemente nesses pacientes. Perda de controle do tônus vascular e alterações estruturais vasculares caracterizadas por proliferação miointimal de pequenas artérias e arteríolas e edema endotelial, levam a uma diminuição do lúmen dos vasos, diminuição do fluxo sangüíneo e a um estado de isquemia crônica ${ }^{(2)}$.

Lesão e disfunção endotelial são também identificadas nas fases precoces da $\mathrm{ES}^{(3,4)}$. Neste contexto, o estresse oxidativo, mediado por radicais livres, leva a uma série de

Disciplina de Reumatologia da Escola Paulista de Medicina, Universidade Federal de São Paulo (UNIFESP). Trabalho recebido em 25/08/05. Aprovado, após revisão, em 03/11/05.

1. Médica doutora associada da disciplina de reumatologia da UNIFESP.

2. Pós-graduanda da disciplina de reumatologia da UNIFESP.

3. Médico residente da disciplina de reumatologia da UNIFESP.

4. Professor Adjunto, livre docente, disciplina de reumatologia da UNIFESP.

Endereço para correspondência: Cristiane Kayser, Rua Botucatu, 740 - $3^{\circ}$ andar, São Paulo, CEP 04023-062, SP, Brasil, e-mail: criskayser@terra.com.br 
disfunções nas células endoteliais e está profundamente envolvido nas alterações vasculares e na patogênese da ES. O estresse oxidativo ocorre quando há um desequilíbrio entre a formação de radicais livres, que pode estar aumentada, e a de antioxidantes, que pode estar diminuída. Os repetidos episódios de isquemia-reperfusão observados na ES, onde o fenômeno de Raynaud (FRy) é a manifestação clínica mais facilmente detectável, acarretam aumento na atividade de radicais livres, com conseqüente lesão e disfunção do sistema endotelial. Postula-se que a prolongada e persistente ativação endotelial que ocorre na ES, induzida pelos episódios de isquemia-reperfusão, leva à perda irreversível da integridade vascular, à lesão tecidual, à ativação de fibroblastos e à ativação do sistema imunológico ${ }^{(5)}$.

Com tantas implicações do estresse oxidativo na patogênese da ES, o uso de drogas antioxidantes tem sido proposto por alguns autores para o tratamento da doença. A N-acetilcisteína é um composto tiólico que, sob forma endovenosa, possui potente ação antioxidante, podendo assim ter efeito benéfico sobre as alterações vasculares da doença. Apenas um estudo foi publicado na literatura avaliando a eficácia e tolerabilidade do tratamento com $\mathrm{N}$-acetilcisteína endovenosa em pacientes com $\mathrm{ES}^{(6)}$.

O objetivo do presente trabalho foi descrever o tratamento com $\mathrm{N}$-acetilcisteína endovenosa em um pequeno grupo de pacientes com diagnóstico de ES e com úlceras isquêmicas de extremidades em nosso meio.

\section{RELATO DOS CASOS}

Três pacientes oriundos do Ambulatório de Doenças do Espectro da Esclerose Sistêmica do Hospital São Paulo da Universidade Federal de São Paulo (UNIFESP) com diagnóstico de ES, segundo os critérios de classificação do ACR (American College of Rheumatology) ${ }^{(7)}$ e com uma ou mais úlceras de extremidades (dígitos ou artelhos) ativas foram tratados com $\mathrm{N}$-acetilcisteína endovenosa. Todos os pacientes apresentavam úlceras isquêmicas que não haviam respondido a tratamento com drogas vasodilatadoras ou inibidores da angiotensina, utilizadas habitualmente em nosso ambulatório.

As principais características demográficas e clínicas dos pacientes encontram-se na Tabela 1.

A posologia e via de administração da $\mathrm{N}$-acetilcisteína baseou-se no esquema utilizado por Sambo et a ${ }^{(6)}$, consistindo na administração de altas doses de $\mathrm{N}$-acetilcisteína sob forma endovenosa contínua por cinco dias. Inicialmente, foi realizada uma dose de ataque de $150 \mathrm{mg} / \mathrm{kg}$ de N-acetil-
TABELA 1

Características Demográficas e Clínicas dos Pacientes Tratados COM N-ACETILCISTÉ́NA SOB Forma Endovenosa

\begin{tabular}{|c|c|c|c|}
\hline Caracteristicas & Paciente 1 & Paciente 2 & Paciente 3 \\
\hline Idade (anos) & 47 & 28 & 25 \\
\hline Sexo & $\mathrm{F}$ & $\mathrm{F}$ & $\mathrm{F}$ \\
\hline Tempo de diagnóstico & 5 anos & 3 anos & 8 anos \\
\hline $\begin{array}{c}\text { Tempo de fenômeno } \\
\text { de Raynaud }\end{array}$ & 6 anos & 7 anos & 19 anos \\
\hline $\begin{array}{l}\text { Forma da doença } \\
\text { (limitada/difusa) }\end{array}$ & difusa & limitada & difusa \\
\hline $\begin{array}{l}\text { Número de úlceras } \\
\text { no início do tratamento }\end{array}$ & 2 & 2 & 3 \\
\hline $\begin{array}{l}\text { Acometimento } \\
\text { pulmonar }\end{array}$ & presente & ausente & presente \\
\hline Acometimento cardíaco & ausente & ausente & ausente \\
\hline $\begin{array}{c}\text { Acometimento } \\
\text { esofagiano }\end{array}$ & presente & ausente & presente \\
\hline Acometimento renal & ausente & ausente & ausente \\
\hline $\begin{array}{l}\text { Escore cutâneo de } \\
\text { Rodnan }\end{array}$ & 25 & 1 & 3 \\
\hline $\begin{array}{c}\text { Presença de } \\
\text { HAP* }\left(\text { PSAP**) }^{\text {(Ecodopplercardiograma) }}\right.\end{array}$ & $\begin{array}{c}\text { Presente } \\
(30 \mathrm{mmHg})\end{array}$ & $\begin{array}{l}\text { ausente (PSAP } \\
\text { não aferida) }\end{array}$ & $\begin{array}{c}\text { Presente } \\
(35 \mathrm{mmHg})\end{array}$ \\
\hline $\begin{array}{l}\text { Outras drogas em uso } \\
\text { durante o tratamento }\end{array}$ & Omeprazol & Carbamazepina & $\begin{array}{c}\text { Prednisona } \\
\text { Cefalexina } \\
\text { AAS } \\
\text { Ranitidina }\end{array}$ \\
\hline
\end{tabular}

*HAP - Hipertensão de artéria pulmonar.

**PSAP - Pressão sistólica de artéria pulmonar.

cisteína diluída em $250 \mathrm{ml}$ de solução salina, administrada em 2 horas. A seguir, a dose foi ajustada para $15 \mathrm{mg} / \mathrm{kg} / \mathrm{h}$ diluída em $1000 \mathrm{ml}$ de solução salina por dia, sob forma de infusão contínua por 5 dias. A infusão foi administrada em ambiente hospitalar, sendo que os pacientes permaneceram internados durante este período.

Para avaliação da eficácia da droga os pacientes foram avaliados nos dias $0,5,15,30$ e 60 pós-infusão. Em todas as avaliações foi contabilizado o número de úlceras, inclusive o aparecimento de novas úlceras, e medida a extensão das mesmas (diâmetro da lesão em milímetros). Em cada avaliação, os pacientes foram também questionados quanto à gravidade do FRy na última semana com uma escala 
visual analógica de gravidade (graduação de 0 a 10 , onde 0 equivale a ausência de episódios de FRy, e 10 , aos piores episódios já experimentados pelo doente), e quanto à intensidade dolorosa do FRy com a escala visual analógica da dor com graduação de 0 a 10 , onde 0 equivale a nenhuma dor, e 10 , à dor extrema.

No início do tratamento, duas pacientes (Paciente l e 2) apresentavam duas úlceras digitais e a paciente 3 , três úlceras em artelhos do membro inferior direito. Progressivamente, foi observada relevante melhora nas dimensões de várias das úlceras, principalmente nas pacientes 1 e 2 , atingindo-se maior grau de melhora após 1 mês do início do tratamento. As pacientes l e 2 apresentaram diminuição importante no diâmetro das duas úlceras após o tratamento, sendo que a paciente 1 apresentou cicatrização completa de uma das úlceras (Tabela 2). Na Figura 1, observamos diminuição importante do diâmetro de uma úlcera digital após 30 dias de tratamento na paciente 2 . Já a paciente 3 apresentou cicatrização de uma úlcera e piora no diâmetro das duas outras úlceras (Tabela 2). Devido a dificuldades de locomoção, a paciente 3 não foi avaliada no dia 30 . Vale salientar que esta paciente apresentava vasculopatia periférica grave, inclusive com amputação do membro inferior esquerdo há aproximadamente dois anos. Ao acompanharmos, a mesma por um período mais prolongado, e após a introdução de nifedipina $60 \mathrm{mg} /$ dia e losartan $100 \mathrm{mg} /$ dia, observamos melhora das úlceras citadas 90 dias após a infusão. Esta melhora tardia não foi computada como resultante do tratamento com a $\mathrm{N}$-acetilcisteína. As pacientes $\mathrm{l}$ e 2 não necessitaram de outras drogas para o tratamento do FRy durante o acompanhamento. Nenhuma das pacientes apresentou novas úlceras durante o acompanhamento.

Ao final de 30 dias, as pacientes 1 e 3 apresentaram melhora importante no FRy, avaliado pela escala visual

TABELA 2

Evolução do Diâmetro (Milímetros) das Úlceras após o Tratamento com N-Acetilcisteína nos Três Pacientes Descritos

\begin{tabular}{c|cc|cc|ccc}
\hline \multirow{2}{*}{$\begin{array}{c}\text { Tempo de } \\
\text { tratamento }\end{array}$} & \multicolumn{2}{|c|}{ Paciente 1 } & \multicolumn{2}{c|}{ Paciente 2 } & \multicolumn{3}{c}{ Paciente 3 } \\
\cline { 2 - 7 } & \multicolumn{7}{c}{ Úlceras } \\
\hline & A & B & A & B & A & B & C \\
Dia 0 & $12 \times 19$ & $10,5 \times 17$ & $10 \times 15$ & $5 \times 4$ & $11 \times 14$ & $3 \times 7$ & $3 \times 4$ \\
Dia 5 & $7 \times 15$ & $6 \times 11$ & $10 \times 11$ & $4 \times 3$ & $15 \times 14$ & $5 \times 7$ & $3 \times 4$ \\
\hline Dia 15 & $4 \times 14$ & $4 \times 8$ & $10 \times 9$ & $3,5 \times 3$ & $22 \times 14$ & $12 \times 14$ & $3 \times 4$ \\
\hline Dia 30 & $4 \times 10$ & $2 \times 7$ & $10 \times 6$ & $3,5 \times 3$ & & & \\
\hline Dia 60 & $4 \times 9$ & $0 \times 0 *$ & $2,5 \times 3$ & $3 \times 3$ & $24 \times 11$ & $11 \times 5$ & $0 \times 0 *$ \\
\hline
\end{tabular}

* * Úlceras que cicatrizaram inteiramente.
Figura 1

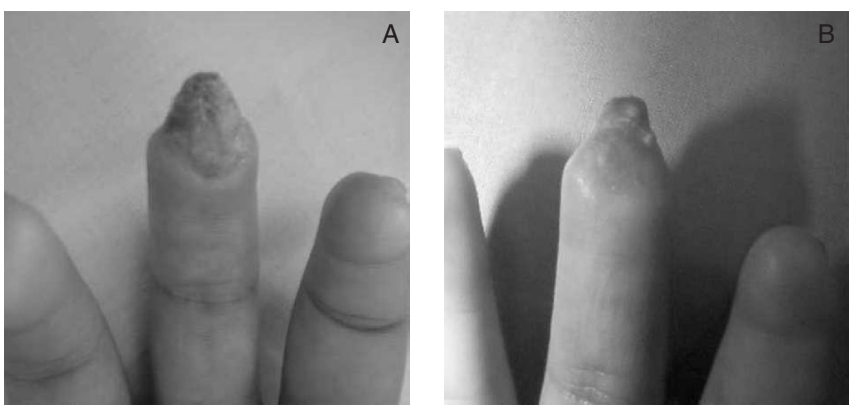

Figura 1 - Evolução de úlcera digital em mão direita no paciente 2 nos dias $0(A)$ e após 30 dias (B) de tratamento com $\mathrm{N}$-acetilcisteína endovenosa.

analógica de gravidade e pela escala visual analógica da dor. Já a paciente 2 não referia presença de FRy no início do tratamento sendo que evoluiu com surgimento de discreto FRy ao final do estudo, referindo apenas uma dor leve associada aos episódios de vasoespasmo (Figura 2).

FIGURA 2
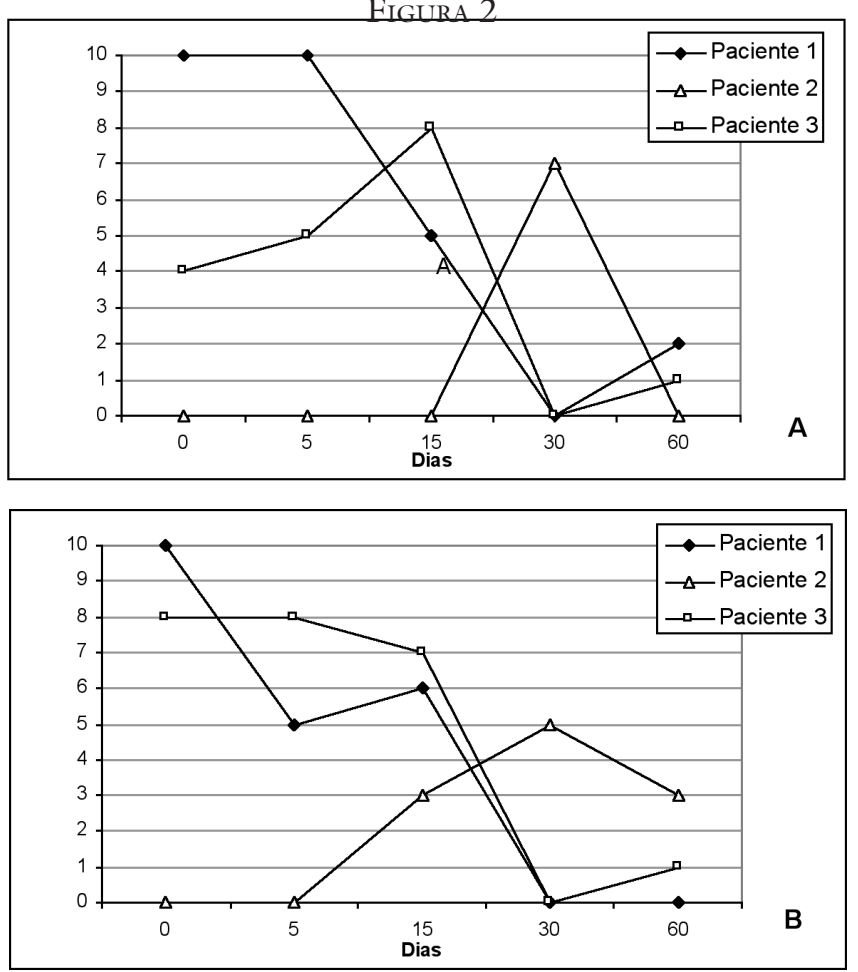

Figura 2 - Evolução da escala visual analógica de gravidade (A) e escala visual analógica da dor (B) durante o acompanhamento nos três pacientes.

\section{DISCUSSÃO}

Não existe até o momento um tratamento eficaz específico para a ES. Para o tratamento de úlceras digitais, o tradicional tratamento utilizado para o FRy com bloqueadores de canal de cálcio (nifedipina) ou inibidores da enzima 
conversora da angiotensina não costuma ser eficaz. Outras drogas, como os análogos da prostaglandina e a bosentana têm se mostrado mais eficazes no tratamento e prevenção de úlceras isquêmicas na $\mathrm{ES}^{(8,9)}$. Entretanto esses dois últimos medicamentos possuem custo extremamente elevado e encontram-se ainda pouco disponíveis em nosso meio.

Devido ao papel que o estresse oxidativo exerce sobre a função endotelial e a patogênese da ES, faz sentido se avaliar a eficácia de drogas antioxidantes no tratamento desta enfermidade, principalmente no sistema vascular.

A N-acetilcisteína é um composto tiólico, com ação mucolítica, utilizado primariamente em doenças obstrutivas e congestivas pulmonares e utilizado mais recentemente no tratamento de SARA e intoxicação por acetaminofeno. Pelas características de sua estrutura química, deduz-se que tenha propriedades antioxidantes, possuindo ação direta como depurador de radicais livres e, por ação indireta, como substrato para a glutationa peroxidase, estimulando a síntese da glutationa, e assim prevenindo a oxidação e degradação do óxido nítrico $(\mathrm{ON})$. Compostos tiólicos e a glutationa são importantes no balanço do estresse oxidativo; níveis diminuídos desses compostos resultam em aumento da oxidação de $\mathrm{ON}$ em nitrato e peroxinitrato ${ }^{(10,11)}$. O peroxinitrato, por sua vez, é um potente oxidante que medeia lesão celular e tecidual em várias condições patológicas ${ }^{(5)}$.

Furst et al ${ }^{12)}$, em 1979, realizaram um estudo duplocego, placebo controlado, com $\mathrm{N}$-acetilcisteína oral em doses convencionais para o tratamento da ES. Nesse estudo, a $\mathrm{N}$-acetilcisteína não levou à melhora da maioria dos parâmetros avaliados (eletrocardiograma, prova de função pulmonar, manometria esofagiana, esofagograma, função renal e acometimento cutâneo) após um ano de uso do medicamento. O fenômeno de Raynaud e a presença de úlceras de extremidades não foram avaliados.

Já que a N-acetilcisteína após administração oral possui baixa biodisponibilidade (menos de 5\%) ${ }^{(13)}$, Sambo et a $\mathbf{l}^{(6)}$, em 2001, desenvolveram um protocolo de tratamento com $\mathrm{N}$-acetilcisteína endovenosa em altas doses, por um período de cinco dias. Os autores realizaram um estudo aberto piloto onde foram avaliadas a eficácia e tolerabilidade do

\section{REFERÊNCIAS}

1. Seibold JR, Smith EA, Leroy C, Steen VD: Systemic sclerosis. In: Klippel JH, Dieppe PA: Rheumatology, London, Mosby-year book Europe limited, 1994.

2. Kahaleh MB: Vascular disease in scleroderma: endothelial $\mathrm{T}$ lymphocyte-fibroblast interactions. Rheum Dis Clin North Am tratamento com $\mathrm{N}$-acetilcisteína endovenosa em pacientes com ES. Este estudo pioneiro mostrou boa tolerabilidade e melhora na freqüência e gravidade do FRy em um grupo de 22 pacientes que receberam a $\mathrm{N}$-acetilcisteína endovenosa. Dos 22 pacientes, 17 apresentavam úlceras isquêmicas de extremidades no início do tratamento (média de 2,94 $\pm 1,63$ úlceras), sendo que houve uma diminuição significativa no número médio de úlceras ao final de 60 dias de acompanhamento (média de 1,53 $\pm 1,64$ úlceras). Observaram também melhora da perfusão digital avaliada através de pletismografia associada a um estímulo frio após o tratamento na maioria dos pacientes.

Além das propriedades antioxidantes, a $\mathrm{N}$-acetilcisteína também parece ter ação vasodilatadora, principalmente na microvasculatura, o que poderia resultar em efeito benéfico sobre os episódios de vasoespasmo nos pacientes com ES. A N-acetilcisteína mostrou ser capaz de melhorar o fluxo sangüíneo da microcirculação em tabagistas e também mostrou ser capaz de melhorar a vasodilatação coronariana e aumentar a dilatação periférica dependente do endotélio em pacientes submetidos a cateterismo cardíaco ${ }^{(10,11)}$.

Todos os três pacientes que descrevemos apresentavam quadro grave de úlceras isquêmicas de extremidades, um dos quais com amputação prévia de um membro. Dois pacientes apresentaram melhora importante das úlceras e os dois que referiam FRy ao início do tratamento também referiram melhora dos eventos vasoespásticos, corroborando os achados do estudo pioneiro de Sambo et a ${ }^{(6)}$. Já o paciente 3, que apresentava um quadro de vasculopatia periférica mais grave, apresentou melhora de apenas uma úlcera.

Este é o primeiro relato no Brasil e o segundo no mundo avaliando o uso da $\mathrm{N}$-acetilcisteína endovenosa em altas doses para o tratamento de úlceras isquêmicas em pacientes com ES. O regime de altas doses endovenosas de $\mathrm{N}$-acetilcisteína mostrou um bom resultado para o tratamento das úlceras nos três pacientes descritos. Esses resultados preliminares positivos legitimam que se conduzam estudos controlados duplo-cegos com placebo para melhor avaliação da eficácia da droga no tratamento das manifestações vasculares e viscerais da ES.
16: 53-73, 1990.

3. Kahaleh MB: The vascular endothelium in scleroderma. Intern Rev Immuno 12: 227-45, 1995.

4. Prescott RJ, Freemont AJ, Jones CJ, Hoyland J, Fielding P: Sequential dermal microvascular and perivascular changes in the development of scleroderma. J Pathol 166: 255-63, 1992. 
5. Herrick AL, Matucci Cerinic M: The emerging problem of oxidative stress and the role of antioxidants in systemic sclerosis. Clin Exp Rheumatol 19: 4-8, 2001.

6. Sambo A, Amico D, Giacomelli R et al: Intravenous $\mathrm{N}$ acetylcysteine for treatment of Raynaud's phenomenon secondary to systemic sclerosis: a pilot study. J Rheumatol 28: 2257-62, 2001.

7. Masi AT, Rodnan GP, Medsger Jr TA et al: Preliminary criteria for the classification of systemic sclerosis (scleroderma). Arthritis Rheum 23: 581-90, 1980.

8. Scorza R, Caronni M, Mascagni B et al: Effects of long-term cyclic iloprost therapy in systemic sclerosis with Raynaud's phenomenon. A randomized, controlled study. Clin Exp Rheumatol 19: 503-8, 2001.

9. Ramos-Casals M, Brito-Zeron P, Nardi N et al: Successful treatment of severe Raynaud's phenomenon with bosentan in four patients with systemic sclerosis. Rheumatology (Oxford)
43: 1454-6, 2004.

10. Andrews NP, Prasad A, Quyyumi AA: N-acetylcysteine improves coronary and peripheral vascular function. J Am Coll Cardiol 37: 117-23, 2001

11. Lu Q, Bjorkhem I, Xiu RJ, Henriksson P, Freyschuss A: Nacetylcysteine improves microcirculatory flow during smoking: new effects of an old drug with possible benefits for smokers. Clin Cardiol 24: 511-5, 2001.

12. Furst DE, Clements PJ, Harris R, Ross M, Levy J, Paulus HE: Measurement of clinical change in progressive systemic sclerosis: a 1 year double-blind placebo-controlled trial of $\mathrm{N}$ acetylcysteine. Ann Rheum Dis 38: 356-61, 1979.

13. Olsson B, Johansson M, Gabrielsson J, Bolme P: Pharmacokinetics and bioavailability of reduced and oxidized $\mathrm{N}$-acetylcysteine. Eur J Clin Pharmacol 34: 77-82, 1988. 